\title{
Surface Dynamics Measurements of Silica Glass
}

\author{
W. Steurer,* A. Apfolter, M. Koch, and W.E. Ernst \\ Institute of Experimental Physics, \\ Graz University of Technology, \\ 8010 Graz, Austria
}

\author{
E. Søndergård \\ Laboratoire Surface et Interface du Verre, \\ UMR 125 CNRS/ Saint-Gobain, \\ 39 Quai Lucien Lefranc, \\ 93303 Aubervilliers Cedex, France
}

\author{
J.R. Manson \\ Department of Physics and Astronomy, \\ Clemson University, Clemson, \\ South Carolina 29634, U.S.A.
}

B. Holst ${ }^{\dagger}$

Institut of Experimental Physics,

Graz University of Technology,

8010 Graz, Austria

(Dated: May 8, 2008) 


\begin{abstract}
We present helium atom scattering experiments from the vitreous silica surface. Time-of-flight data of scattered He-atoms have been recorded at varying temperature and as a function of parallel momentum transfer. A dominant elastic peak and two broad features at $\approx 4 \mathrm{meV}$, one each on the phonon annihilation and creation side, are observed in all spectra. The thermal measurements between 127.0 and 368.5 K unveil clear Debye-Waller attenuation behavior of the elastic peak and indicate that scattering occurs in the single-phonon regime below $200 \mathrm{~K}$. The root mean square vibration amplitude at room temperature is estimated to be $0.014 \mathrm{~nm}$ with a corresponding surface Debye temperature of $340 \pm 20 \mathrm{~K}$ and an effective mass of 18 a.u. The obtained values agree well with bulk values under the assumption of force constants at the surface reduced by one-half. The wavevector dependence of the Debye-Waller exponent indicates a well depth of the atomsurface interaction potential of $10 \pm 2 \mathrm{meV}$. The broad inelastic features on the phonon creation and annihilation sides are identified as the surface manifestation of the boson peak phenomenon in amorphous materials. An unusual shifting of the boson peak modes to higher energies with increasing temperature is observed. Measurements at different parallel momentum transfers unveil a non-dispersive behavior of the boson peak mode.
\end{abstract}

PACS numbers: 61.43.Fs,63.50.+x,78.30.Ly,72.10.Di

Keywords: Boson peak, vitreous silica, glass 


\section{INTRODUCTION}

Surface properties are of paramount importance for many macroscopic glass properties such as durability and ductility. This becomes apparant in the process of cutting sheet glass by scribing its surface with a diamond tip. The hard and brittle material breaks along a sharp line upon applied pressure with similarities to cleaving a crystal. But this is already where the commonness of glasses and crystalline materials ends. Glasses not only differ from crystals in that they lack long-range order, the level of understanding of their surface properties - surface dynamics and structure in particular - is still rudimentary relative to crystalline materials. Whereas ordered surfaces have been the subject of intense research for more than 40 years and especially metal surfaces are well understood nowadays, even the surface structure of the most important pristine glass, vitreous silica, is only vaguely known. The reasons for the comparably little knowledge might be manifold but are at least partly due to the fact that diffraction methods are hindered by the missing translational symmetry of the surface which prohibits the definition of a finite unit cell. Furthermore, direct imaging techniques such as atomic force microscopy and scanning tunneling microscopy fail in obtaining high-resolution images for the technologically important melt-formed surface condition.

Experimental evidence on the nanoscale structure of the silica glass surface are scant and currently available achievements will be discussed in Sect. . But even less is known about the surface dynamics of vitreous silica. Efforts on shedding light onto surface dynamics of silica glass have extended to model calculations already many years ago. With growing computer power, molecular dynamics simulations have become valuable tools that are capable of predicting bond angle distributions, ring sizes, anion distributions, etc., as well as estimating $\mathrm{OH}$ concentrations as a result of hydroxylation of the silica glass surface [1-6]. Furthermore, the density of states of surface vibrations has been calculated, predicting an excess of states at energies $<10 \mathrm{meV}[2,3]$. Excess modes over a Debye density of states, a phenomenon commonly termed "boson peak" (BP) [7, 8], have until now only been observed in bulk measurements. Recently, the BP has been discovered as a surface phenomenon by the authors of this paper in qualitative agreement with the predictions [9].

In the course of the experiment a chopped beam of He-atoms impinged on the surface and time-of-flight (TOF) data of the scattered particles were recorded. At low beam energies 
as used for the experiments here, the He projectiles are non-penetrating and are reflected from the repulsive part of the atom-surface interaction potential several atomic units above the centers of the first layer of surface atoms. The obtained spectra feature a dominant elastic peak and two inelastic peaks on the phonon creation side and phonon annihilation side, respectively, as shown in Fig. 1. The inelastic features appear as excess modes over the Debye limit in the extracted surface phonon spectral density and are thus attributed to the surface manifestation of the BP phenomenon. The approach of extracting a surface phonon spectral density from TOF records has been presented in Ref. [9]. It is derived from a theory of atom-surface scattering in the kinematic approximation [10] under the assumption that one-phonon processes are dominant and that multi-phonon scattering is negligible.

Here we present thermal attenuation measurements of the silica glass surface by helium atom scattering and give evidence that at low temperatures, such as used in Refs. [9, 11, 12], scattering is indeed in the single phonon regime. If the elastic intensity decays exponentially with increasing temperature (see Fig. 2) and the Debye-Waller exponent is small (less than one), then the interaction is clearly in the single-phonon regime since the Debye-Waller exponent corresponds roughly to the number of phonons created or destroyed in a surface collision. We present a detailed analysis of the thermal attenuation measurements using Debye theory from which a mean-square vibration amplitude, an effective mass as well as a surface Debye temperature are estimated.

The paper is organized as follows. In Sect. the experimental procedures are described and a list of all experiments performed is provided. The theoretical approach of extracting the surface phonon spectral density is recapitulated in Sect. . In the first part of Sect. the elastic peak intensity of the thermal measurements is analyzed. Justification is given that at low temperatures scattering occurs predominantly in the single-phonon scattering regime. The second part of Sect. focuses on the inelastic features seen in the TOF records. A compilation of previously published BP results is presented as well as further analysis of the data from Ref. [9]. The results obtained are then discussed in Sect. . Finally, conclusions are presented in Sect. . 


\section{EXPERIMENTAL PROCEDURES}

A polished Spectrosil@ glass sample (780 ppm bulk OH content), produced by hydrolyzation of $\mathrm{SiCl}_{4}$ when spraying into an oxygen-hydrogen flame [13], was used in the present experiment. The sample had dimensions $10 \times 10 \times 1 \mathrm{~mm}^{3}$ and underwent an elaborate cleaning procedure which has already been described in Ref. [11]. The clean sample was transferred under an argon atmosphere into a transport chamber and was shipped from Saint Gobain to the helium scattering apparatus in Graz [14]. The sample was mounted onto the sample holder while keeping it permanently under an argon atmosphere, similar to the procedure described in Ref. [15]. For temperature control, a K-type thermocouple was spot welded next to the sample. The thermocouple was calibrated by venting the chamber and placing an additional thermocouple on top of the sample after the experiments had been completed. Before starting experiments the surface was exposed to an initial cleaning in situ [15]. Similar to the $\alpha$-quartz (0001) surface [15] the surface of silica glass maintained a constant He-reflectivity at room temperature over a period of days without any further cleaning, thus indicating that it is extremely inert against contamination.

The experiments were performed at a base pressure in the $10^{-9}$ mbar range. A nearly monochromatic beam was created by supersonic expansion through a nozzle with different energies (between 20 and $30 \mathrm{meV}$ ) for the individual data sets. An energy spread of $\delta E / E \approx$ $2 \%$ was characteristic for all data sets obtained. The scattered helium atoms were ionised by electron bombardment, sent through a magnetic mass selector and detected by a channeltron. The detector entrance was $1618 \mathrm{~mm}$ from the sample surface. Experiments were performed for several beam energies and setup configurations as listed in Table I.

\section{ATOM-SURFACE SCATTERING FORMALISM}

The basic elements of surface scattering theory follow methods that have been introduced in studying neutron or x-ray scattering from bulk solids [16]. All quantities measurable in a scattering event can be obtained from the generalized Fermi golden rule

$$
w_{f i}=\left\langle\frac{2 \pi}{\hbar} \sum_{\left\{n_{f}\right\}}\left|\mathcal{T}_{f i}\right|^{2} \delta\left(\mathcal{E}_{f}-\mathcal{E}_{i}\right)\right\rangle
$$


Table I: Recorded He-scattering data sets from the silica glass surface. The beam energy $E_{i}$ remained constant while either the surface temperature $T_{S}$ or the parallel momentum transfer was changed. The latter depends on beam energy and incident and final angle, $\theta_{i}$ and $\theta_{f}$, respectively. All spectra were sampled over a total recording time $t_{m}$ at a given bin width $t_{b} . N$ is is the number of spectra recorded for each data set.

\begin{tabular}{|c|c|c|c|c|c|c|c|c|}
\hline Data set & description & $E_{i}(\mathrm{meV})$ & $T_{S}(\mathrm{~K})$ & $\theta_{i}(\operatorname{deg})$ & $\theta_{f}(\mathrm{deg})$ & $t_{m}(\mathrm{~s})$ & $t_{b}(\mu \mathrm{s})$ & $N$ \\
\hline 1 & Debye-Waller & 26.6 & $128.3-256.6$ & 30 & 60 & 500 & 5 & 4 \\
\hline 2 & attenuation & 20.6 & $128.4-256.6$ & 40 & 40 & 500 & 5 & 5 \\
\hline 3 & measurements & 28.0 & $128.2-256.6$ & 40 & 40 & 500 & 5 & 5 \\
\hline 4 & & 31.7 & $128.2-256.5$ & 40 & 40 & 500 & 5 & 5 \\
\hline 5 & & 26.5 & $128.3-256.5$ & 40 & 50 & 500 & 5 & 5 \\
\hline 6 & & 21.5 & $128.7-256.6$ & 45 & 45 & 500 & 5 & 5 \\
\hline 7 & & 24.6 & $127.2-283.0$ & 45 & 45 & 500 & 5 & 5 \\
\hline 8 & & 25.2 & $129.7-256.6$ & 45 & 45 & 500 & 5 & 5 \\
\hline 9 & & 28.9 & $129.1-283.0$ & 45 & 45 & 500 & 5 & 6 \\
\hline 10 & & 27.9 & $129.9-256.5$ & 45 & 45 & 500 & 5 & 6 \\
\hline 11 & & 29.9 & $128.2-256.6$ & 45 & 45 & 500 & 5 & 5 \\
\hline 12 & & 32.2 & $129.0-256.6$ & 45 & 45 & 500 & 5 & 5 \\
\hline 13 & & 21.6 & $128.5-256.5$ & 60 & 30 & 500 & 5 & 5 \\
\hline 14 & & 19.9 & $127.0-368.5$ & 45 & 45 & 2000 & 2.5 & 9 \\
\hline 15 & $\begin{array}{l}\text { Varying parallel } \\
\text { momentum transfer }\end{array}$ & 20.0 & 127.0 & 45 & $33-53$ & 2000 & $2.5^{a}$ & 11 \\
\hline
\end{tabular}

${ }^{a}$ The bin width given in Ref. [9] refers to the effective bin width after data treatment.

where the average over initial target states is denoted by the angular brackets \langle\rangle and the sum is over all unmeasured final states $\left\{n_{f}\right\}$ of the target which can scatter a projectile into its specified final state. The energies $\mathcal{E}_{i}$ and $\mathcal{E}_{f}$ refer to the total energy of the system of projectile molecule plus the target before and after collision, respectively. The $\mathcal{T}_{\text {fi }}$ are the matrix elements of the transition operator $\mathcal{T}$ taken with respect to unperturbed initial and final states of the system. The transition rate is the fundamental quantity for describing a 
scattering process, because all measurable quantities can be calculated from it.

In the semiclassical limit, and assuming linear response in the displacements of the target surface, the transition rate can be expressed as the Fourier transform over all times and directions parallel to the surface of a generalized time-dependent correlation function

$$
\begin{aligned}
w_{f i}= & \frac{1}{\hbar^{2}}\left|\tau_{f i}\right|^{2} \int_{-\infty}^{\infty} d t e^{-i\left(E_{f}-E_{i}\right) t / \hbar} \\
& \times \int_{-\infty}^{\infty} d \mathbf{R} e^{i \mathbf{K} \cdot \mathbf{R}} e^{-2 \mathcal{W}\left(\mathbf{k}_{f}, \mathbf{k}_{i}\right)} e^{Q(\mathbf{R}, t)} .
\end{aligned}
$$

where $\exp \left\{-2 \mathcal{W}\left(\mathbf{k}_{f}, \mathbf{k}_{i}\right)\right\}$ is the Debye-Waller factor and $Q(\mathbf{R}, t)$ is a generalized time and position dependent correlation function. At this level of approximation $\left|\tau_{f i}\right|^{2}$ is the scattering form factor and $\tau_{f i}$ is the off-energy-shell transition matrix element of the elastic part of the interaction potential. The relation between the Debye-Waller argument and the generalized correlation function is $2 \mathcal{W}\left(\mathbf{k}_{f}, \mathbf{k}_{i}\right)=Q(\mathbf{R}=0, t=0)$.

A simple but effective approximation is to assume that the collision with the surface is rapid compared to a typical phonon period, in which case the semiclassical force exerted on the scattering particle is replaced by the momentum impulse and the correlation function becomes the time and position dependent displacement correlation function

$$
Q(\mathbf{R}, t)=\langle\mathbf{k} \cdot \mathbf{u}(0,0) \mathbf{k} \cdot \mathbf{u}(\mathbf{R}, t)\rangle
$$

where $\mathbf{u}(\mathbf{R}, t)$ is the phonon displacement at the position $\mathbf{R}$ on the surface and $\mathbf{k}=\mathbf{k}_{f}-\mathbf{k}_{i}$ is the scattering vector. The argument of the Debye-Waller factor is then given by

$$
\mathcal{W}\left(\mathbf{k}_{f}, \mathbf{k}_{i}\right)=\left\langle(\mathbf{k} \cdot \mathbf{u}(0,0))^{2}\right\rangle
$$

Equation 2 provides a standard and very convenient method for developing the scattering transition into an ordered series in numbers of phonons transferred in the collision process by expanding the exponentiated correlation function in powers of its argument $Q(\mathbf{R}, t)$. Expansion to first order leads to the approximation of the single phonon contribution

$$
\begin{aligned}
w_{f i}= & \frac{2 \pi}{\hbar}\left|\tau_{f i}\right|^{2} e^{-2 \mathcal{W}(\mathbf{k})} \\
& \times\left\{\begin{array}{l}
n(\omega) \\
n(\omega)+1
\end{array}\right\} \mathbf{k} \cdot \underline{\underline{\rho(\mathbf{K}, \omega)}} \cdot \mathbf{k} .
\end{aligned}
$$

The Bose-Einstein functions, $n(\omega)$ and $n(\omega)+1$, are for annihilation and creation events, respectively. The surface phonon spectral density $\underline{\underline{\rho(\mathbf{K}, \omega)}}$ is a tensor of rank two and function 
of frequency $\omega$ and parallel momentum transfer $\mathbf{K}$ which is essentially the surface projection of the phonon density of states and is given by [17]:

$$
\begin{array}{r}
\rho_{\alpha, \alpha^{\prime}}(\mathbf{K}, \omega)=\sum_{\nu} \frac{\hbar}{2 \omega_{\nu}(\mathbf{K}) M} \\
\times e_{\alpha}(\mathbf{K}, \nu) e_{\alpha^{\prime}}(\mathbf{K}, \nu) \delta\left(\omega-\omega_{\nu}(\mathbf{K})\right),
\end{array}
$$

where $\omega_{\nu}(\mathbf{K})$ is the phonon frequency depending on parallel wave vector and other quantum numbers $\nu, M$ is the effective surface mass, $\alpha$ and $\alpha^{\prime}$ are indices that run over the three cartesian directions and $e_{\alpha}(\mathbf{K}, \nu)$ is the phonon polarization vector.

The experimental quantity which is usually measured in the case of He-scattering from surfaces is the differential reflection coefficient $d^{3} R\left(\mathbf{k}_{f}, \mathbf{k}_{i}\right) / d \Omega d E_{f}$ giving the fraction of incident particles scattered into a small solid angle of $d \Omega$ and an energy interval $d E_{f}$. This is obtained from the transition rate by dividing by the incident flux crossing a plane parallel to the surface and multiplying by the density of final particle states

$$
\frac{d^{3} R}{d \Omega_{f} d E_{f}}=\frac{L^{4}}{(2 \pi \hbar)^{3}} \frac{m^{2}\left|\mathbf{k}_{f}\right|}{k_{i z}} w_{f i},
$$

where $L$ is a quantization length and $m$ is the particle mass.

Equation 7 is the starting point for the derivation of the spectral density from the measured TOF record. The differential reflection coefficient is directly proportional to the number of counts measured in the experiment. If, with the exception of $\underline{\underline{\rho}}$, expressions for each of the factors appearing in Eq. (7) are determined then $\underline{\underline{\rho}}$ can be extracted through comparison with sufficient experimental data.

The final wavevector $\mathbf{k}_{f}$ is determined by the experimental setup and the scattering amplitude $\tau_{f i}$ is approximated by the Morse matrix element $[18,19]$, i.e., the matrix element of $V(r)=D\left(1-e^{-\beta z}\right)^{2}$ taken with respect to its proper wave functions where $z$ is the direction perpendicular to the surface. A value of $D=10 \mathrm{meV}$ is chosen for the well depth of the Morse potential according to the value gained from the analysis of the thermal attenuation data presented below in Sect. . The range parameter is chosen to be $\beta=$ $25 \mathrm{~nm}^{-1}$, a value that is appropriate for insulator surfaces [20]. The Morse matrix element is an improvement relative to the approximation made in Ref. [9] where a Mott-Jackson matrix element [19, 21] was used. The Debye-Waller exponent is used in an approximate expression which accounts for the $\mathbf{k}$-dependence observed in the thermal attenuation measurements presented in Sect. below, $2 \mathcal{W} \propto C_{1} \mathbf{k}^{2} T+C_{2} T$ where $C_{1}$ and $C_{2}$ are constants depending 
on surface Debye temperature, well depth and effective mass which have been determined from the thermal attenuation measurements. In the single quantum limit $\hbar \omega=\left|E_{f}-E_{i}\right|$ and the Bose-Einstein distribution $n(\omega)$ reduces to $k_{B} T /\left|E_{f}-E_{i}\right|$ for $\hbar \omega \ll k_{B} T$. In the experiments presented here the parallel momentum transfer $\mathbf{K}$ is more than an order of magnitude smaller than the perpendicular momentum transfer $\Delta k_{z}$ so for both the DebyeWaller factor of Eq. (4) and the transition rate of Eq. (5) it can be neglected, thus the experiment is preferentially sensitive only to the normal surface spectral density element $\rho_{z z}(\omega, \mathbf{K})$. After all the above approximations the spectral density $\rho_{z z}$ (written for the annihilation side) is given by

$$
\rho_{z z} \propto \frac{\left[\frac{d R\left(\mathbf{k}_{f}, \mathbf{k}_{i}\right)}{d \Omega d E_{f}}\right]^{\text {expt. }}\left|E_{f}-E_{i}\right|}{\left|\tau_{f i}\right|^{2} E_{f}^{3 / 2} \exp \left\{-C_{1} \mathbf{k}^{2} T+C_{2} T\right\}},
$$

where the superscript expt. denotes that the differential reflection coefficient is that obtained from experiment. The linear term in $\left|E_{f}-E_{i}\right|$ is the characteristic signature for the small frequency region of Debye model behavior in the surface phonon spectral density.

The precondition for this transformation is that the interaction of the He-projectile with the surface is in the single-phone regime. Indications for being in this regime come from excluding the contrasting case of multiple-phonon interaction $[22,23]$ which would be characterized by a temperature dependent increase of the width of the inelastic (multiphonon) background which goes approximately according to the power law $T^{1 / 2}$, and from a clear Debye-Waller behavior of the elastic peak intensity with the argument $2 W<1$. If the elastic intensity decays exponentially with increasing temperature and the Debye-Waller exponent is small, then the interaction is clearly in the single-phonon regime since the Debye-Waller exponent roughly corresponds to the number of phonons created or destroyed in a surface collision.

\section{RESULTS}

A typical energy-converted TOF record is depicted in Fig. 1. All spectra feature a dominant elastic peak (FWHM $\approx 2 \mathrm{meV}$ ) above an intense inelastic background. Some smaller structure (marked by short arrows in Fig. 1 ) is seen in the inelastic part on the phonon annihilation and creation sides. The higher noise level on the phonon creation side of the 


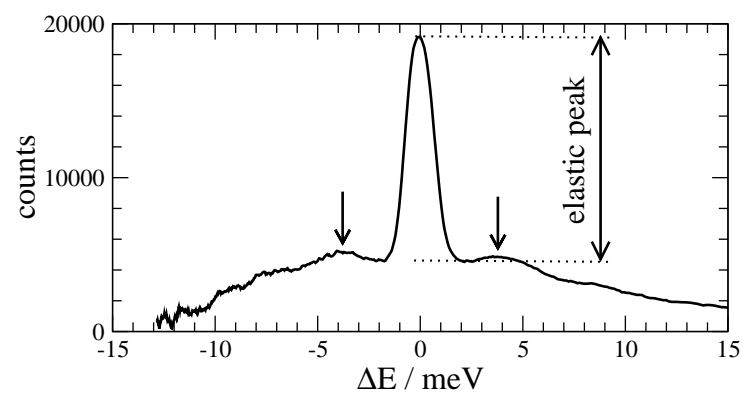

Figure 1: Typical time-of-flight record converted to an energy transfer scale for the abscissa and to the differential reflection coefficient for the ordinate. A dominant elastic peak (FWHM $\approx 2 \mathrm{meV})$ is featured by all records. The short arrows point at the inelastic features which are identified as the surface manifestation of the boson peak phenomenon. The depicted record is taken from data set No. $14 ; T=137.2 \mathrm{~K}, \Theta_{f}=45^{\circ}, E_{i}=19.9 \mathrm{meV}$.

energy-resolved spectra shown in Fig. 1 is an artifact due to the Jacobian in the conversion of the data from TOF to energy transfer.

\section{Elastic peak attenuation}

The intensity of the elastic peak can be extracted from an energy-converted TOF spectrum by a least-squares fit of a sum of $N$ Lorentzian functions ( $N$ depends on the individual spectrum, typically 3 to 5) and retaining only the intensity of the dominant amplitude. In Fig. 2 the elastic peak intensity is shown in a semi-logarithmic plot versus sample temperature for the data sets No. 1 to No. 14 from Table I. The depicted intensities decrease exponentially with increasing temperature according to

$$
I(T)=I_{0} e^{-2 W(T)}
$$

where $I_{0}$ is the intensity from a lattice at rest and $\exp [-2 W(T)]$ is the Debye-Waller factor. The upper scale on the abscissa in Fig. 2 depicts the Debye-Waller argument $2 W$ which is obtained from the slopes of the exponential fits. At temperatures below $200 \mathrm{~K}$ the argument $2 W<1$ and, therefore, scattering occurs clearly in the single-phonon regime at these conditions. For small momentum transfers parallel to the surface Eq. (4) can be approximated by 

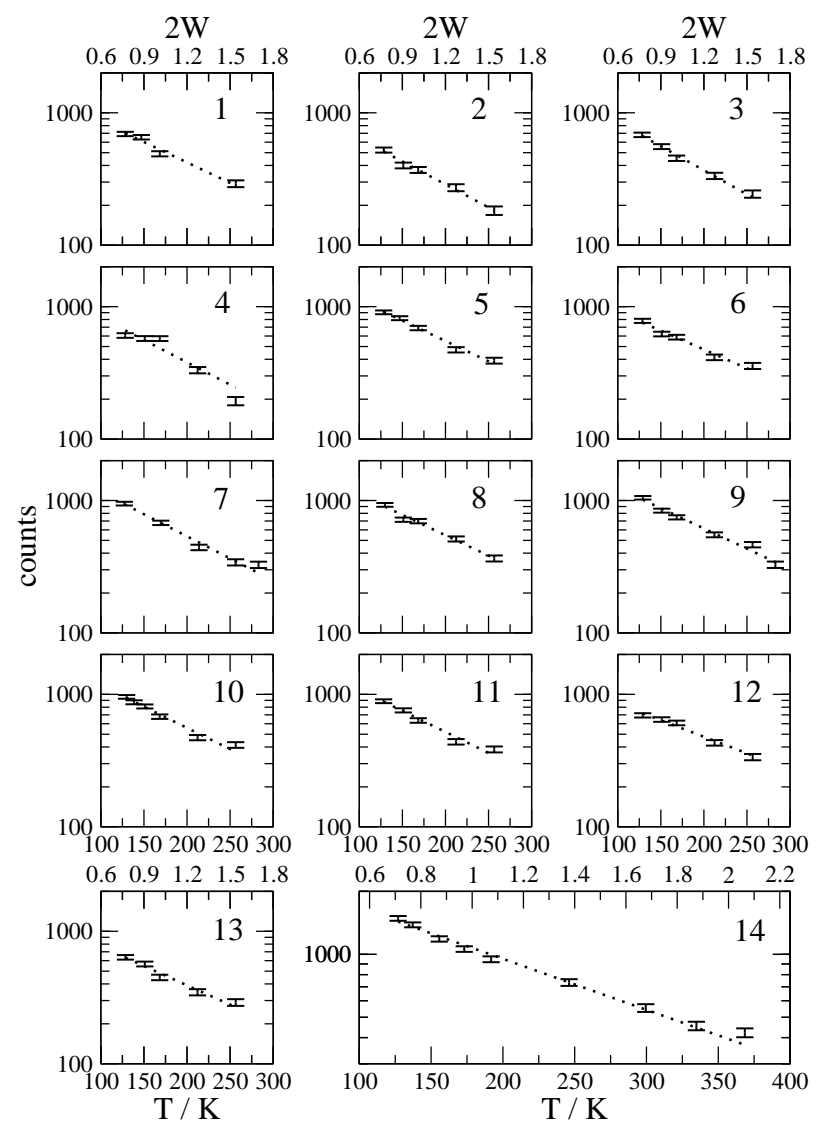

Figure 2: Logarithmic depiction of the Debye-Waller attenuation measurements for the data sets No. 1 to No. 14 from Table I. The lower scale of the abscissa depicts the sample temperature whereas the upper scale shows the Debye-Waller argument $2 W$ (same scaling for data sets No. 1 to No. 13). The dotted lines are exponential fits to the data.

$$
2 W(T)=\left\langle u_{z}^{2}\right\rangle\left(\Delta k_{z}\right)^{2}
$$

where $\left\langle u_{z}^{2}\right\rangle$ is the average displacement of a crystal atom perpendicular to the surface. Equation 10 is strictly valid only for the specular beam, however, it is also a reasonable approximation for final angles not too different from the incident angle. If the surface phonons can be described by a Debye distribution, then the Debye-Waller exponent takes the simple form $[24]$

$$
2 W(T)=\frac{3 \hbar^{2} \Delta k_{z}^{2} T}{M k_{B} \Theta_{D}^{2}}
$$


where $M$ is the mass of a surface atom, $k_{B}$ is the Boltzmann constant and $\Theta_{D}$ is the surface Debye temperature. In case of materials made up of more than just one element, $M$ has to be replaced by an effective mass $M_{e f f}$. The effect of the attractive well near the surface can be taken into account by replacing $\Delta k_{z}$ by

$$
\Delta k_{z}^{\prime}=\left(k_{f z}^{2}+2 m D / \hbar^{2}\right)^{1 / 2}+\left(k_{i z}^{2}+2 m D / \hbar^{2}\right)^{1 / 2}
$$

where $m$ is the particle mass, $D$ the well depth of the helium-surface interaction potential, and the superscripts $f$ and $i$ stand for final and incident wavevector, respectively. This approximation is called the "Beeby correction" [25] and has been successfully applied to many Debye-Waller attenuation studies in the past. Finally,

$$
2 W(T)=\frac{3\left(\hbar^{2} \Delta k_{z}^{2}+8 m D\right) T}{M k_{B} \Theta_{D}^{2}},
$$

if the momentum transfer parallel to the surface is zero or small compared to the perpendicular momentum transfer as is the case for the measurements presented here. Equation 13 identifies the coefficients $C_{1}$ and $C_{2}$ appearing in Eq. (8).

In Fig. 3, the Debye-Waller argument $2 W(T)$ is expressed as

$$
2 W(T)=C_{1} \Delta \mathbf{k}^{2} T
$$

and the constant $C_{1}$ is depicted versus $\Delta \mathbf{k}^{2}$, showing a $\Delta \mathbf{k}$ dependence as is observed for many materials [26]; the dotted line is drawn as a guide to the eye.

From the observed $\Delta \mathbf{k}^{2}$ dependence the well depth of the helium-surface interaction potential can be obtained through a three-parameter fit to the data $\left[D,\left(M_{\text {eff }} \Theta_{D}^{2}\right)\right.$ and $\left.I_{0}\right]$, as follows from inserting Eq. (13) in Eq. (9). A value of $D=10 \pm 2 \mathrm{meV}$ is obtained this way for the well depth of the atom-surface interaction potential in good agreement with the potential well depth found for the helium- $\alpha$-quartz system [27].

The second fit parameter $\left(M_{e f f} \Theta_{D}^{2}\right)$ is a product of two quantities which, in principle, cannot be separated in a straight-forward manner. However, comparison of Eq. (10) and Eq. (14) yields a mean vibration amplitude of a surface atom of $\left\langle u_{z}^{2}\right\rangle=2.1 \cdot 10^{-4} \mathrm{~nm}^{2}$ at room temperature that is roughly a factor two larger than the bulk value $\left\langle u_{z}^{2}\right\rangle=1 / 3\left\langle\mathbf{u}^{2}\right\rangle=$ $0.95 \cdot 10^{-4} \mathrm{~nm}^{2}$ (at $300 \mathrm{~K}$ ) reported by Nakamura et al. [28]. The observed factor of two is well understood for a simple model where the force constants acting on a surface atom in the normal direction are reduced by one-half compared to atoms in the bulk. Within 


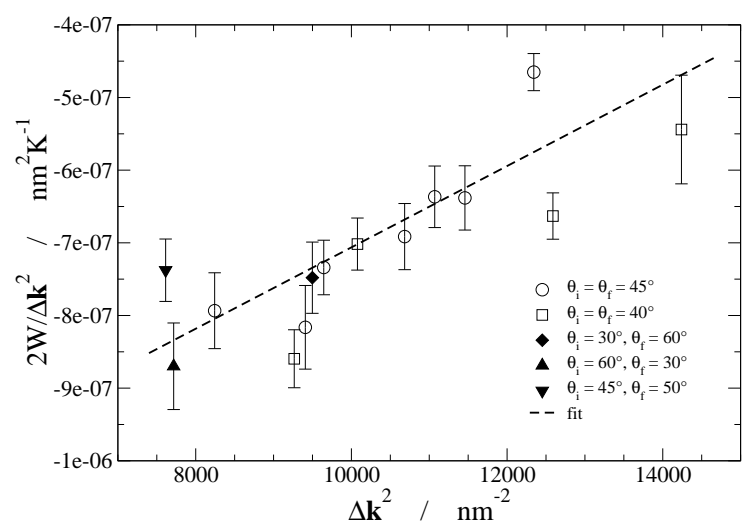

Figure 3: Measured Debye-Waller exponents versus perpendicular momentum transfer squared showing the usual $k$-dependence.

this simple model, the surface Debye temperature is related to the bulk Debye temperature by $[29]$

$$
\Theta_{D, \text { surface }}=\frac{1}{\sqrt{2}} \Theta_{D, \text { bulk }} .
$$

Thus, the good agreement of the measured mean-squared vibration amplitudes of an atom at the surface compared to an atom in the bulk indicates that Eq. (15) yields a good estimate for the surface Debye temperature. For a bulk Debye temperature of $494 \mathrm{~K}$ [7] (calculated from sound velocities [55] ) a surface Debye temperature of $\approx 350 \mathrm{~K}(=1 / \sqrt{2} \cdot 494 \mathrm{~K})$ would thus be expected. For $\Theta_{D}=350 \mathrm{~K}$ the effective mass $M_{\text {eff }}$ is estimated from the second fit parameter, yielding 18 a.m.u.

This is seen to be a reasonable value. The silica surface is generally accepted to terminate with hydroxyl groups [30,31] and, furthermore, investigations by atomic force microscopy show that silicon atoms are rarely located at the outermost surface layer [32]. The effective mass of 18 a.m.u. is slightly smaller than the effective mass which was found in elastic peak attenuation measurements from the $\alpha$-quartz (0001) surface [27].

\section{Excess modes}

In Fig. 1, broad peaks are seen in the inelastic part of the spectrum on both the phonon annihilation and creation sides. The observed features at $\approx \pm 4 \mathrm{meV}$ (marked by short arrows in Fig. 1) are observed in all spectra. In extracted surface phonon spectral densities, these peaks appear as excess modes over the Debye density of states and are thus attributed 


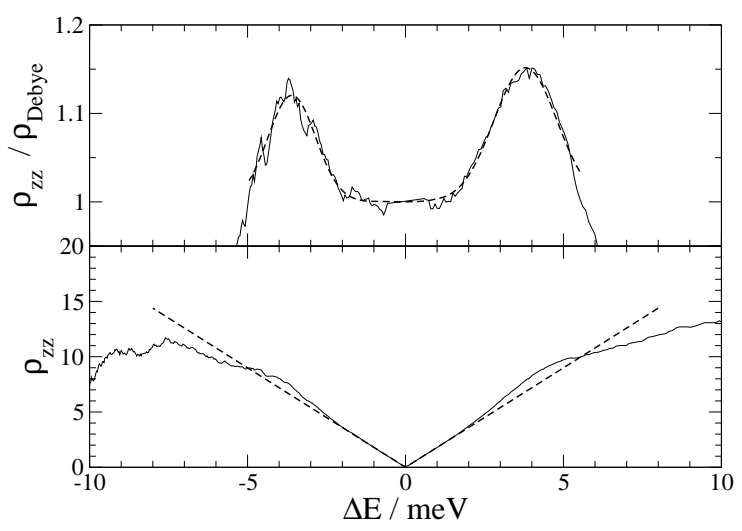

Figure 4: Lower panel: An excess contribution to states compared to a Debye model (dashed line) is seen in the experimentally obtained surface phonon spectral density (from data set No. 14, TOF record obtained with the sample at $137.2 \mathrm{~K}$ ). Upper panel: The BP is best seen in a depiction of measured spectral density divided by the Debye limit, $\rho_{z z} / \rho_{\text {Debye }}$.

to the surface manifestation of the BP phenomenon. A typical extracted surface phonon spectral density is shown in Fig. 4.

A sequence of surface phonon spectral densities, extracted from TOF records at varying parallel momentum transfers, is shown in Fig. 5 as a depiction of $\rho_{z z} / \rho_{\text {Debye }}$. The respective measurements (data set No. 15 from Table I) were obtained by changing the final beam angle while all other parameters remained fixed [9]. Figure 5 clearly reveals the non-dispersive nature of the BP. It is noted, however, that the creation and annihilation peaks for the spectra in Fig. 5 are not identical, contrary to what is expected on the basis of the detailed balance condition. Since the different probabilities for annihilation and creation events have already been factored out in the analysis, the imprecision must be due to approximations in the analysis. The most likely of these approximations is the use of a Born approximation matrix element of the Morse potential to calculate the form factor in Eq. (8), while another cause may be neglect of parallel momentum contributions. The solid line drawn for each plot in Fig. 5 is a least-squares fit to the extracted surface phonon spectral density by the sum of two Gaussian functions. From the fit parameters, the energy of the BP modes can be determined more accurately than from the TOF spectra, $\Delta E=3.7 \pm 0.4 \mathrm{meV}[11]$. In anticipation of the observed temperature dependence of the BP which will be discussed in the next paragraph, it is important to note that these measurements were performed with the sample at $127.0 \mathrm{~K}$. No clear dependence upon the parallel momentum transfer rate is 


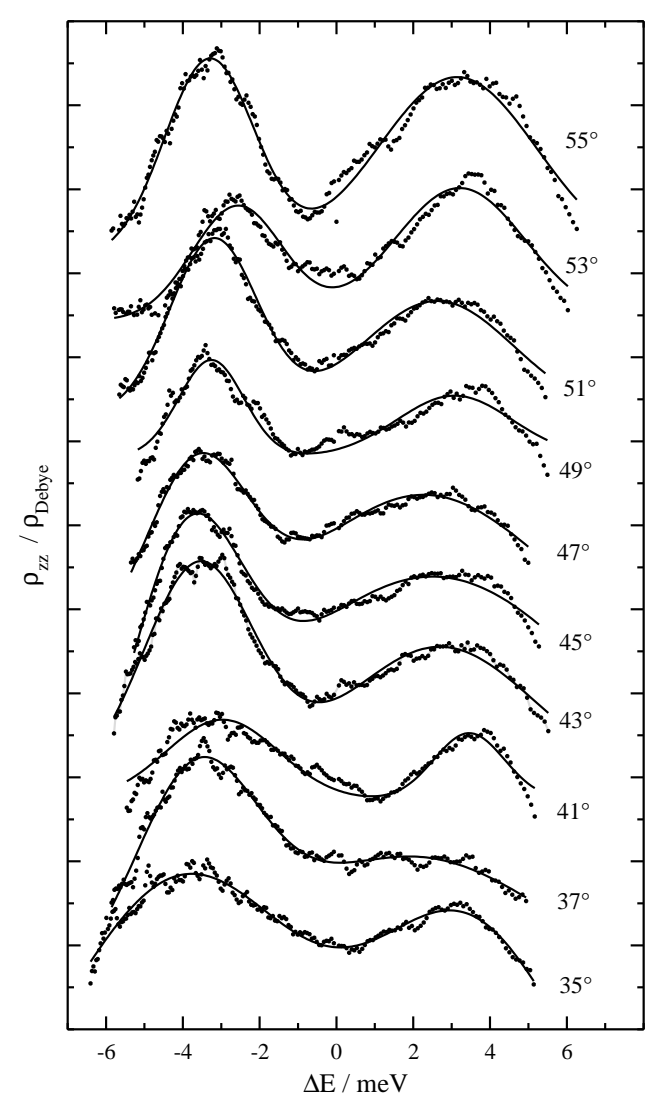

Figure 5: Representation of the boson peak data at varying parallel wavevector transfer (data set No. 15 from Table I, $T=127.0 \mathrm{~K})$ in $\rho_{z z} / \rho_{\text {Debye }}$ versus $\Delta E$; values for the final beam angle $\theta_{f}$ are shown next to each plot. Individual spectra are shifted by 0.1 along the ordinate; the scale of the ordinate has 0.02 spacing.

obtained for the width of the BP [11], yielding a mean FWHM value of $2.6 \pm 0.6 \mathrm{meV}$. In contrast, an interesting dependence upon the parallel momentum transfer is seen for $\rho_{z z}(\Delta E=$ const., $\Delta \mathbf{K}) / \rho_{\text {Debye }}$ as depicted in Fig. 6. The apparent maximum in the neighborhood of $1 \mathrm{~nm}^{-1}$ may lend further support to the highly-discussed question of the "end of the acoustic branch" [33, 34]. A further peculiarity of the experiments at varying parallel momentum transfers is the absence of a Rayleigh peak in the TOF spectra which has been reported in Fig. 1 of Ref. [9]. At low beam energies, such as used in all experiments presented herein, a Rayleigh mode would be expected for a flat surface. An explanation for the absence of the Rayleigh mode has been suggested by the authors of this paper based on a particular surface structure which is made up of small areas with slopes of up to $7^{\circ}[9]$. 


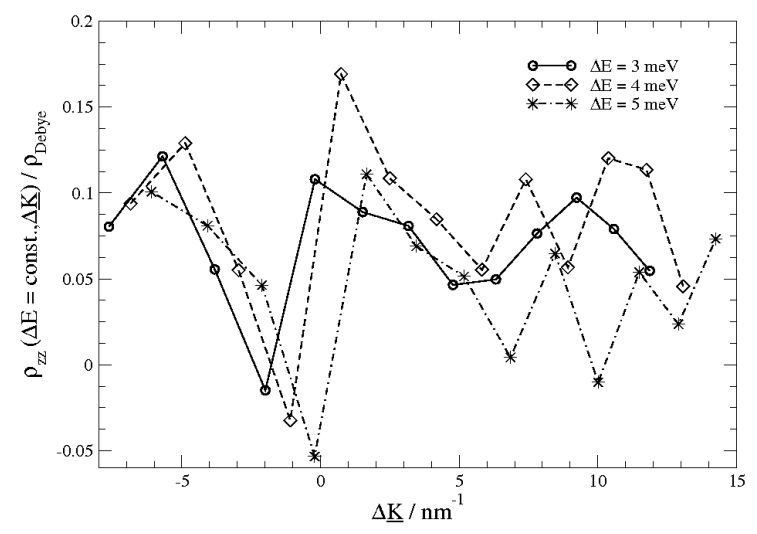

Figure 6: Intensity of the BP at constant energy as a function of the parallel momentum transfer, from Ref. [11].

A more extensive discussion of the implications of the surface dynamics measurements for resolving the surface structure of silica glass is presented in Sect. . For a more detailed description and analysis of the dispersionless nature of the BP we refer to Refs. [9, 11].

In addition to the elastic peak intensity of the thermal data (data sets No. 1 to No. 14) which has already been analyzed in Sect. , the inelastic features that are seen on the phonon annihilation and creation side unveil an anomalous temperature dependence of the surface BP. With increasing temperature, the most probable energy of the surface BP in silica glass shifts to higher energies [12] contrary to what is usually expected for phonon modes as a consequence of normal anharmonic behavior. An anomalous positive energy shift is observed in bulk silica, but the observed shifting of the surface BP is surprisingly strong compared to measurements of the BP in bulk silica glass by inelastic neutron scattering [3537], Raman scattering [36, 38, 39], Brillouin scattering [40] and inelastic x-ray scattering experiments [40], as shown in Fig. 7. By extracting a surface phonon spectral density from the TOF records of data set No. 14 a shift rate of $0.08 \mathrm{meV} / \mathrm{K}$ is obtained which is a factor of four to ten stronger than shift rates in the bulk.

\section{DISCUSSION}

\section{Surface boson peak}

The measurements of the surface boson peak add a further set of observations to the experimental characterization of the BP phenomenon. In the case of the surface sensitive 


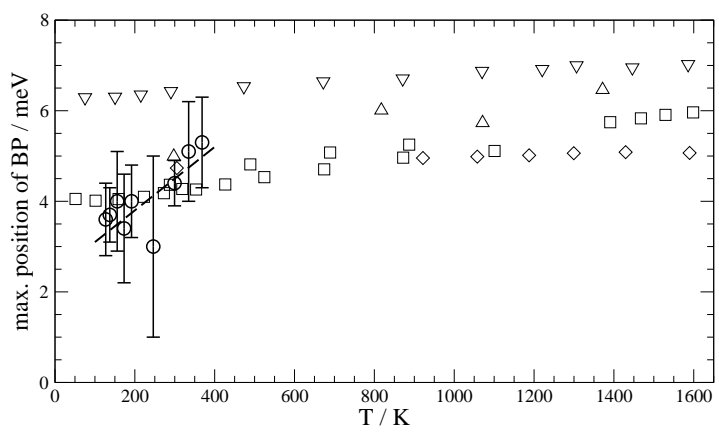

Figure 7: Plot of the most probable energy of the BP versus temperature as measured by the different investigation techniques: helium atom scattering $(O)$, inelastic neutron scattering $[37](\square)$, Raman scattering [39] $(\nabla)$, inelastic x-ray scattering [40] $(\triangle)$, and Brillouin light scattering [40] $(\diamond)$. The errorbars on the helium scattering data points indicate FWHM of the surface BP.

measurements by helium atom scattering, properties of the BP can be accessed in a straightforward fashion by extraction of the surface phonon spectral density from the TOF records. Our measurements unveil

- clear non-dispersive behavior of the surface BP

- apparent oscillations in the amplitude of the BP at constant energy as a function of parallel momentum transfer, i.e., $\rho_{z z}(\Delta E=$ const., $\Delta \mathbf{K}) / \rho_{\text {Debye }}=f(\Delta \mathbf{K})$

- no clear dependence of the width of the surface BP upon temperature and parallel momentum transfer

- a strong temperature dependence of the most probable energy of the surface BP, shifting to higher energies with increasing temperature.

The dispersionless nature of the surface BP is in contrast to bulk measurements by inelastic X-ray scattering and inelastic neutron scattering where a dispersive BP has been observed [34, 40,41]. A highly debated topic in this context are speculations about an "end of the acoustic" branch. This concept implies that the description of phonons by plane waves should loose its validity beyond a certain wavevector where plane waves cease to exist. In densified silica, the limiting wavevector nearly coincides with the BP maximum [33, 42, 43]. However, the topic is still a matter of debate since experimental evidence of such a crossover has not yet been obtained for vitreous silica [34]. The oscillations seen in Fig. 6 may lend 
further support to these speculations since the putative maximum in the neighborhood of $1 \mathrm{~nm}^{-1}$ could arise due to such an effect. Yet, the number of data points is not sufficiently dense as to make any serious statements in this direction at the moment.

The strong temperature dependence of the surface BP compared to that in the bulk cannot be explained satisfactorily yet. In Ref. [12] the authors of this paper have suggested that the anomalous shift direction has the same origin as the unusual temperature dependence of the bulk modulus of silica glass. The shifting of the BP to higher energies with increasing temperature implies a high negative Grüneisen parameter. At moderate temperatures such as in the experiments presented here, the negative Grüneisen parameter arises due to the anomalous temperature dependence of the elastic bulk modulus which has been observed recently in Brillouin spectroscopy experiments [44, 45]. Hence, with increasing temperature the structure of silica glass becomes more rigid and, as a consequence, the excess modes shift to higher energies with increasing temperature.

\section{Surface structure}

As already mentioned in the introduction, there are not many experimental investigations of the silica glass surface. High-resolution atomic-force microscopy images of silica glass surfaces have up till now only been obtained for surfaces created by fracture in UHV conditions [32, 46-48]. Features such as interatomic distances and groupings of atoms have been seen in images reported by Poggemann et al. [46] Successive works [47, 48] have unveiled clusters at the fracture surface of size $>1 \mathrm{~nm}$ in accordance with the model of preordered regions as proposed by Brückner [49]. Recently, Raberg et al. have reported correlation measurements by atomic force microscopy on fractured silicate glasses [32]. Their investigations confirmed the role of $\mathrm{SiO}_{4}$ tetrahedra as the basic building blocks but showed also the existence of some destroyed or incomplete tetrahedra at the surface. In contrast, investigations of melt-formed silica glasses by atomic force microscopy $[50,51]$ have been reported only for rather large scan sizes in the range of $500 \times 500 \mathrm{~nm}^{2}$. Different origins for the intrinsic roughnesses of melt-formed and fractured surfaces have been proposed on the basis of a comparative study of analyzing RMS values [50]. The roughness of the pristine melt-formed surface is controlled by the surface tension of the melt surface and can quantitatively be described by frozen capillary waves [51]. On the other hand, it is proposed that 
the minimum observed roughness for fracture surfaces in the mirror zone reflects the bulk structure of glass.

Although helium atom scattering has been proposed as a tool for resolving the structure of silica glass on the nanometer scale more than twenty years ago [52], new insight into the surface structure of fused silica glass (i.e., melt-formed, as opposed to the fractured surfaces of Poggemann et al.) is gained by the measurements presented here.

- The measured mean-square vibration amplitudes are roughly a factor two larger than vibration amplitudes measured in the bulk.

- The assumption of reduced force constants at the surface by one-half in the direction perpendicular to the surface leads to an effective mass $M_{e f f}=18$ a.m.u., thus confirming the hydroxyl-terminations at the silica surface.

- Estimated well depths for the helium-silica surface interaction potential and the helium- $\alpha$-quartz surface interaction potential are almost identical.

- The Rayleigh wave is not seen in the data sets obtained at varying parallel momentum transfer. This peculiarity can be explained by assuming a surface structure that is made up of slopes which are tilted at various small angles.

Whereas the measured mean-square vibration amplitudes are consistent with measurements in the bulk by inelastic neutron scattering [28], only qualitative agreement is obtained between molecular dynamics simulations [4] and the measurements presented here. The reported mean-square displacement values by Wang et al. are a factor of two larger than measured values at the surface of silica glass and in the bulk [28]. Only qualitative agreement with our measurements is achieved in that a factor of two between mean-square displacement values for the surface and the bulk was predicted.

The absence of the Rayleigh mode at large wavelengths for the silica glass surface cannot be fully explained yet. An analysis of the diffuse elastic peak intensity observed in the energy-resolved TOF spectra [53] indicates that the glass surface exhibits a roughness at the $3 \mathrm{~nm}$ scale with a distribution of slopes of up to $7^{\circ}$ with respect to the surface plane. For parallel wave vectors corresponding to wavelengths shorter than $3 \mathrm{~nm}$, a given scan curve would intersect the dispersion curve from all slopes within the coherence region of 
the incident He atom beam, thus smearing out the signal due to the Rayleigh mode and making it unobservable. Only for wavelengths significantly larger than the characteristic size of $3 \mathrm{~nm}$ would a clear Rayleigh mode be observable, and that corresponds to parallel momentum vectors of order $0.2 \mathrm{~nm}^{-1}$ or less which is below the sensitivity of the present experiment.

\section{CONCLUSIONS}

We have presented helium scattering experiments from the silica glass surface. Thermal attenuation measurements of the elastic peak show clear Debye-Waller behavior. The DebyeWaller argument is $<1$ at temperatures below $200 \mathrm{~K}$ indicating that scattering occurs in the single-phonon regime at these temperatures. The predominance of one-phonon processes at the surface of silica glass is a precondition for the extraction of the surface phonon spectral density from recorded TOFs. The inelastic features seen in the TOF records appear as densities in excess over a Debye model of the surface phonon spectral density and are therefore identified as the surface manifestation of the BP phenomenon. The surface BP is observed as a dispersionless mode. An unusual shift direction of BP to higher energies with increasing temperature is seen in the thermal data.

\section{Acknowledgements}

The authors gratefully acknowledge support from the European Commission, FP6, NEST STREP ADVENTURE program, Project INA, contract number 509014. W.S. is supported by a DOC-fellowship of the Austrian Academy of Science.

* Electronic address: wolfram.steurer@gmail.com

$\dagger$ Present Address: Department of Physics and Technology, University of Bergen, Allegaten 55, 5007 Bergen, Norway

[1] M. I. Trioni, A. Bongiorno, and L. Colombo, J. Non-Cryst. Solids 220, 164 (1997).

[2] M. Wilson and T. R. Walsh, J. Chem. Phys. 113, 9180 (2000).

[3] C. Wang, Y. Tamai, and N. Kuzuu, J. of Non-Cryst. Solids 321, 204 (2003). 
[4] C. Wang, N. Kuzuu, and Y. Tamai, Journal of Non-Crystalline Solids 318, 131 (2003).

[5] E. A. Leed and C. G. Pantano, Journal of Non-Crystalline Solids 325, 48 (2003).

[6] J. Du and A. Cormack, J. Am. Ceram. Soc. 88, 2978 (2005).

[7] T. Nakayama, Rep. Prog. Phys. 65, 1195 (2002).

[8] S. Yannopoulos, K. Andrikopoulos, and G. Ruocco, J. Non-Cryst. Solids 352, 4541 (2006).

[9] W. Steurer, A. Apfolter, M. Koch, W. E. Ernst, B. Holst, E. Søndergård, and J. R. Manson, Phys. Rev. Lett. 99, 035503 (2007).

[10] J. R. Manson, Phys. Rev. B 43, 6924 (1991).

[11] W. Steurer, A. Apfolter, M. Koch, W. E. Ernst, E. Søndergård, J. R. Manson, and B. Holst, J. Phys.: Condens. Matter (2008), in press.

[12] W. Steurer, A. Apfolter, M. Koch, W. E. Ernst, E. Søndergård, J. R. Manson, and B. Holst, Phys. Rev. Lett. 100, 135504 (2008).

[13] R. Brückner, J. Non-Cryst. Solids 5, 123 (1970).

[14] A. Apfolter, Master's thesis, Graz University of Technology (2005), unpublished.

[15] W. Steurer, A. Apfolter, M. Koch, T. Sarlat, E. Søndergård, W. Ernst, and B. Holst, Surf. Sc. 601, 4407 (2007).

[16] A. A. Maradudin, E. W. Montroll, and G. H. Weiss, Theory of Lattice Dynamics in the Harmonic Approximation (New York, Academic Press, 1963).

[17] V. Celli, Dynamic Properties of Solids (Elsevier, New York, 1990).

[18] A. R. Devonshire, Proc. of the Royal Society (London) A 158, 269 (1937).

[19] F. O. Goodman and H. Y. Wachman, Dynamics of Gas-Surface Scattering (Academic Press, New York, 1976).

[20] F. Hofmann, J. P. Toennies, and J. R. Manson, J. Chem. Phys. 101, 10155 (1994).

[21] J. M. Jackson and N. F. Mott, Proc. Royal Society (London) A 137, 703 (1932).

[22] J. R. Manson, Comp. Phys. Comm. 80, 145 (1994).

[23] F. Hofmann, J. P. Toennies, and J. R. Manson, Surf. Sc. 349, L184 (1996).

[24] J. R. Manson, M. Li, and A. P. Graham, J. Phys.: Condens. Matter 14, 6233 (2002).

[25] J. L. Beeby, J. Phys. C 4, L359 (1971).

[26] D. Farias and K.-H. Rieder, Rep. Prog. Phys. 61, 1575 (1998).

[27] W. Steurer, A. Apfolter, M. Koch, W. E. Ernst, and B. Holst, Surf. Sc. 602, 1080 (2008).

[28] M. Nakamura, M. Arai, Y. Inamura, T. Otomo, and S. M. Bennington, Phys. Rev. B 66, 
$024203(2002)$.

[29] F. C. M. J. M. van Delft, Surf. Sc. 251-252, 690 (1991).

[30] O. Sneh and S. M. George, J. Phys. Chem 99(13), 4639 (1995).

[31] B. P. Feuston and S. H. Garofalini, J. Appl. Phys. 68, 4830 (1990).

[32] W. Raberg, A. H. Ostadrahimi, T. Kayser, and K. Wandelt, J. Non-Cryst. Solids 351, 1089 (2005).

[33] E. Rat, M. Foret, E. Courtens, R. Vacher, and M. Arai, Phys. Rev. Lett. 83, 1355 (1999).

[34] M. Foret, R. Vacher, E. Courtens, and G. Monaco, Phys. Rev. B 66, 024204 (2002).

[35] A. Wischnewski, U. Buchenau, A. J. Dianoux, W. A. Kamitakahara, and J. L. Zarestky, Phys. Rev. B 57, 2663 (1998).

[36] A. Fontana, F. Rossi, S. Caponi, E. Fabiani, U. Buchenau, and A. Wischnewski, J. Non-Cryst. Solids 351, 1928 (2005).

[37] A. Wischnewski, Ph.D. thesis, University of Düsseldorf (1998).

[38] G. Winterling, Phys. Rev. B 12, 2432 (1975).

[39] A. Kalampounias, S. Yannopoulos, and G. Papatheodorou, J.Non-Cryst. Solids 352, 4619 (2006).

[40] C. Masciovecchio, V. Mazzacurati, G. Monaco, G. Ruocco, T. Scopigno, F. Sette, P. Benassi, A. Cunsolo, A. Fontana, M. Krisch, et al., Phil. Mag. 79, 2013 (1999).

[41] M. Nakamura, M. Arai, T. Otomo, Y. Inamura, and S. M. Bennington, J. Non-Cryst. Solids 293-295, 377 (2001).

[42] M. Foret, E. Courtens, R. Vacher, and J.-B. Suck, Phys. Rev. Lett. 77, 3831 (1996).

[43] D. A. Parshin and C. Laermans, Phys. Rev. B 63, 132203 (2001), cond-mat/0007462.

[44] A. Polian, D. Vo-Thanh, and P. Richet, Europhys. Lett 57, 375 (2002).

[45] R. Le Parc, C. Levelut, J. Pelous, B. Martinez, and B. Champagnon, J. Phys.: Condens. Matter 18, 7507 (2006).

[46] J. F. Poggemann, A. Goß, G. Heide, E. Radlein, and G. H. Frischat, J. Non-Cryst. Solids 281, 221 (2001).

[47] J. F. Poggemann, G. Heide, and G. H. Frischat, J. Non-Cryst. Solids 326-327, 15 (2003).

[48] G. H. Frischat, J. F. Poggemann, and G. Heide, J. Non-Cryst. Solids 345-346, 197 (2004).

[49] R. Brü ckner, Glastech. Ber. 37, 536 (1964).

[50] P. K. Gupta, D. Inniss, C. R. Kurkjian, and Q. Zhong, J. Non-Cryst. Solids 262, 200 (2000). 
[51] T. Sarlat, A. Lelarge, E. Søndergård, and D. Vandembroucq, Europ. Phys. J. B 54, 121 (2006).

[52] C. H. L. Goodman, Phys. Chem. Glasses 27, 27 (1986).

[53] W. Steurer et al., unpublished results.

[54] U. Buchenau, M. Prager, N. Nücker, A. J. Dianoux, N. Ahmad, and W. A. Phillips, Phys. Rev. B 34, 5665 (1986).

[55] An inconsistency between the value obtained from sound velocities and the value $\Theta_{D, b u l k}=$ $370 \mathrm{~K}$ measured by neutron scattering measurements has been reported by Buchenau et al. [54], however, the origin of this discrepancy has not yet been resolved. 